\title{
Recent successes of cancer immunotherapy: a new dimension in personalized medicine?
}

\author{
Christophe Caux $\cdot$ Laurence Zitvogel
}

Received: 10 January 2012 / Accepted: 18 January 2012 /Published online: 15 February 2012

(C) Springer-Verlag 2012

Until recently, cancer therapy was limited to "debulking approaches" including surgery, chemo-radio- and hormono-therapy and compounds targeting oncogenes or proangiogenic pathways. Unfortunately, phase III studies pointed out that such strategies, remarkable against primary tumors, mostly affected time to progression but rarely overall survival, suggesting that long-term protective effects were not achieved. Immunotherapy which has long been opposed to chemotherapy because of its susceptibility to drug-induced apoptosis and its lack of efficacy to immediately "debulk," comes of age for a main reason: immunotherapy may be the sole approach to elicit cancer-specific adaptive immune responses and long-term protection against metastases. Can it be any cure without the involvement of the immune system?

Cancer immunotherapy currently involves several complementary strategies: immune cell transfer, vaccines, cytokines,

\section{Caux}

Université Lyon 1, ISPB,

69008 Lyon, France

C. Caux $(\bowtie)$

Cancer Research Center of Lyon (CRCL) UMR INSERM 1052 /

CNRS 5286, LabEx DEVweCAN,

Lyon, France

e-mail: christophe.caux@lyon.unicancer.fr

C. Caux

Centre Léon Bérard,

69008 Lyon, France

L. Zitvogel

Center of Clinical Investigations, INSERM U1015,

Institut Gustave Roussy (IGR),

Villejuif, France

L. Zitvogel

University of Paris Sud,

Villejuif, France vaccine adjuvants, and monoclonal antibodies (mAb) endowed with antibody-dependent cell cytotoxicity or interfering with immune checkpoints. Several of these approaches are reviewed in this issue of Targeted Oncology.

Important clinical benefits were first demonstrated using adoptive transfer of autologous tumor-infiltrating lymphocytes resulting in objective cancer regression in a significant proportion of patients with metastatic melanoma. In particular, the combination of cell transfer with a lymphodepleting regimen induced complete durable responses in $40 \%$ of patients, with complete responses ongoing beyond 3 to 7 years. Current cell transfer therapy approaches using autologous cells genetically engineered to express conventional or chimeric $\mathrm{T}$ cell receptors have mediated cancer regression in patients with metastatic melanoma, synovial sarcoma, neuroblastoma, and refractory lymphoma. Adoptive cell transfer immunotherapy is a rapidly developing new approach to the therapy of metastatic cancer in humans and has evolved towards the administration of "young TILs" [1,2].

Recently, the US Food and Drug Administration approved two novel immunotherapy agents, one based on a cancer vaccine (Sipuleucel-T, Dendreon) in patients with indolent hormone refractory prostate cancer and the other one based on targeting a pivotal $\mathrm{T}$ cell inhibitory receptor (anti-CTLA4 mAb, ipilimumab/Yervoy, BMS) in metastatic melanoma patients, which provide a survival benefit in two phase III clinical trials [3, 4].

These encouraging clinical results will be followed by many expected breakthroughs, according to the current evaluations worldwide. These include:

1. Therapeutic vaccines capable of inducing robust $T$ cell responses, using long overlapping peptides or recombinant poxviruses or synthetic mRNA [5-7].

2. Different classes of immune adjuvants such as Toll-likereceptor agonists currently under clinical trial in [8]. 
Those agonists are now undergoing extensive clinical investigations both as immuno-stimulant molecules and as adjuvant along with vaccines.

3. T cell growth factors such as IL-7 capable of reconstituting a diverse immune repertoire in lymphopenic cancer patients and boosting anti-tumor specific immunity [9].

However, it is increasingly documented that tumors develop diverse strategies leading to escape tumor-specific immunity and that immunosuppression associated with cancer has to be overcome to allow a better immunostimulation [10]. Among the specific and active tumor-immunosuppressive mechanisms, $\mathrm{CD} 4^{+} \mathrm{CD} 25^{\text {high }} \mathrm{T}$ regulatory cells (Treg) are important mediators of active immune evasion in cancer [11]. Treg depletion improves endogenous anti-tumor immunity and the efficacy of active immunotherapy in animal models of cancer, suggesting that inhibiting Treg function could also improve the limited successes of human cancer immunotherapy [12]. Specific strategies are currently under development that target Tregs [13].

Combination of immunotherapy with conventional treatments (chemotherapy, radiotherapy, antiangiogenic, etc.) should further improve this approach both in its effectiveness and in its clinical indications. Indeed, accumulating evidence indicate that the innate and adaptive immune systems make a crucial contribution to the anti-tumor effects of conventional chemotherapy- and radiotherapy-based cancer treatments $[14,15]$.

Importantly, to further improve these encouraging clinical results, biomarkers need to be discovered not only to establish correlates with clinical successes but also to further select those patients likely to benefit from immunotherapy. Furthermore, the intricate dialog between tumor molecular hallmarks and immune reactions or disorders need to be delineated to establish the most relevant criteria defining the susceptibility to develop a cancer and/or to respond to a given therapy [16].

In conclusion, the recent positive results of clinical trials with novel immunoactive drugs as well as the unexpected finding of a positive interaction between immunotherapy and chemotherapy should open a new era for the personalized immunotherapy of cancer.

Conflict of interest The authors declare no conflict of interest.

\section{References}

1. Rosenberg SA (2011) Cell transfer immunotherapy for metastatic solid cancer-what clinicians need to know. Nat Rev Clin Oncol 8:577-585

2. Jotereau $\mathrm{F}$, Gervois $\mathrm{N}$, Labarrière $\mathrm{N}$ Adoptive transfer with high affinity TCR to treat human solid tumours: how to improve the feasibility? Targeted Oncol, this issue doi:10.1007/s11523-012$0207-\mathrm{z}$

3. Sharma P, Wagner K, Wolchok JD, Allison JP (2011) Novel cancer immunotherapy agents with survival benefit: recent successes and next steps. Nat Rev Cancer 11:805-812

4. Lesterhuis WJ, Haanen JB, Punt CJ (2011) Cancer immunotherapy-revisited. Nat Rev Drug Discov 10:591-600

5. Melief CJ, van der Burg SH (2008) Immunotherapy of established (pre)malignant disease by synthetic long peptide vaccines. Nat Rev Cancer 8:351-360

6. Boon T, Coulie PG, Van den Eynde BJ, van der Bruggen P (2006) Human $\mathrm{T}$ cell responses against melanoma. Annu Rev Immunol 24:175-208

7. Kantoff PW, Schuetz TJ, Blumenstein BA, Glode LM, Bilhartz DL, Wyand M, Manson K, Panicali DL, Laus R, Schlom J, Dahut WL, Arlen PM, Gulley JL, Godfrey WR (2010) Overall survival analysis of a phase II randomized controlled trial of a poxviralbased PSA-targeted immunotherapy in metastatic castrationresistant prostate cancer. J Clin Oncol 28:1099-1105

8. Goutagny N, Estornes Y, Hasan U, Lebecque S, Caux C Targeting pattern recognition receptors in cancer immunotherapy. Targeted Oncol, this issue doi:10.1007/s11523-012-0209-x

9. Morre M, Beq S Interleukin-7 and immune reconstitution in cancer patients: a new paradigm for dramatically increasing overall survival. Targeted Oncol, this issue doi:10.1007/s11523-012-0210-4

10. Zou W (2005) Immunosuppressive networks in the tumour environment and their therapeutic relevance. Nat Rev Cancer 5:263274

11. Sakaguchi S (2004) Naturally arising CD4+ regulatory t cells for immunologic self-tolerance and negative control of immune responses. Annu Rev Immunol 22:531-562

12. Colombo MP, Piconese S (2007) Regulatory-T-cell inhibition versus depletion: the right choice in cancer immunotherapy. Nat Rev Cancer 7:880-887

13. Ménétrier-Caux C, Curiel T, Faget F, Manuel M, Caux C and Zou $\mathrm{W}$ Targeting regulatory $\mathrm{T}$ cells. Targeted Oncol, this issue doi:10.1007/s11523-012-0208-y

14. Zitvogel L, Apetoh L, Ghiringhelli F, Kroemer G (2008) Immunological aspects of cancer chemotherapy. Nat Rev Immunol 8:5973

15. Zitvogel L, Kepp O, Kroemer G (2011) Immune parameters affecting the efficacy of chemotherapeutic regimens. Nat Rev Clin Oncol 8:151-160

16. Ogino S, Galon J, Fuchs CS, Dranoff G (2011) Cancer immunology-analysis of host and tumor factors for personalized medicine. Nat Rev Clin Oncol 8:711-719 